\title{
The Argonaute family: tentacles that reach into RNAi, developmental control, stem cell maintenance, and tumorigenesis
}

\author{
Michelle A. Carmell, ${ }^{1,2,3}$ Zhenyu Xuan, ${ }^{1,3}$ Michael Q. Zhang, ${ }^{1}$ and Gregory J. Hannon ${ }^{1,4}$ \\ ${ }^{1}$ Cold Spring Harbor Laboratory, Cold Spring Harbor, New York 11724, USA; ${ }^{2}$ Program in Genetics, State University of New \\ York at Stony Brook, Stony Brook, New York 11794, USA
}

RNA interference (RNAi) is an evolutionarily conserved process through which double-stranded RNA (dsRNA) induces the silencing of cognate genes (for review, see Bernstein et al. 2001b; Carthew 2001). Sources of dsRNA silencing triggers include experimentally introduced dsRNAs, RNA viruses, transposons, and RNAs transcribed from complex transgene arrays (for review, see Hammond et al. 2001b). Short hairpin sequences encoded in the genome also appear to enter the RNAi pathway and function to regulate the expression of endogenous, protein-coding genes /Grishok et al. 2001; Hutvagner et al. 2001; Ketting et al. 2001; Knight and Bass 2001; Hutvagner and Zamore 2002).

According to the current mechanistic model, dsRNAs initiate RNAi following their conversion into small, 2124-nucleotide (nt) short interfering RNAs (siRNAs; Hamilton and Baulcombe 1999; Zamore et al. 2000; Bernstein et al. 2001a; Elbashir et al. 2001). The siRNAs then guide an effector complex referred to as the RNAinduced silencing complex, or RISC, to its cognate substrates (Hammond et al. 2000; Zamore et al. 2000). Both biochemical and genetic studies have led to the identification of two, conserved gene families that are universal components of the interference process. These are the Dicer family, which is comprised of members of the Ribonuclease III family of enzymes, and the Argonaute gene family, which is comprised of proteins with unknown biochemical function. An examination of the phenotypes arising from mutations in these classes of genes has revealed that RNAi and related pathways may participate in a rich array of biological processes, many of which are, as yet, only tenuously linked to RNAi. The purpose of this review is to draw from studies of the Argonaute gene family to illustrate the potentially conserved impact of RNA interference and related pathways on diverse biological processes.

\footnotetext{
${ }^{3}$ These authors contributed equally to this work.

${ }^{4}$ Corresponding author.

E-MAIL hannon@cshl.org; FAX (516) 367-8874.

Article and publication are at http://www.genesdev.org/cgi/doi/10.1101/ gad.1026102.
}

\section{The Argonaute family}

Argonaute proteins make up a highly conserved family whose members have been implicated in RNAi and related phenomena in several organisms. In addition to roles in RNAi-like mechanisms, Argonaute proteins influence development, and at least a subset are involved in stem cell fate determination. Argonaute proteins are $\sim 100-\mathrm{kD}$ highly basic proteins that contain two common domains, namely PAZ and PIWI domains (Cerutti et al. 2000). The PAZ domain, consisting of 130 amino acids, has been identified in Argonaute proteins and in Dicer (Bernstein et al. 2001a). Although it has no defined function, PAZ is thought to be a protein-protein interaction domain, potentially mediating both homo- and heterodimerization (Cerutti et al. 2000). In fact, two Drosophila Argonautes, dAgol and dAgo2, coimmunoprecipitate with Dicer (Hammond et al. 2001a; Caudy et al. 2002). However, it is not yet clear whether such interactions depend upon the PAZ domains. The C-terminal 300-amino acid PIWI domain also has no known function, but is highly conserved. Argonaute proteins can be separated according to sequence into two subclasses; those that resemble Arabidopsis AGO1, and those that more closely resemble Drosophila Piwi (Fig. 1).

There are several reports that Argonaute proteins, being highly basic, might bind RNA. Miwi, a murine homolog of Piwi, exists in a complex with its target mRNAs in vivo (Deng and Lin 2002). Furthermore, Drosophila Agol has been shown to bind ribohomopolymers in vitro (Kataoka et al. 2001). Of course, the involvement of Argonaute proteins in RNAi and related phenomena presupposes an interaction with RNA, either directly or indirectly. However, detailed studies of Argonaute structure and biochemistry will be required to reveal its precise function in RNA metabolism.

\section{Argonautes: roles in silencing phenomena and development}

Argonaute family genes have been isolated from several organisms in screens for mutants that are deficient in RNAi and related phenomena, including post-transcrip- 
Carmell et al.

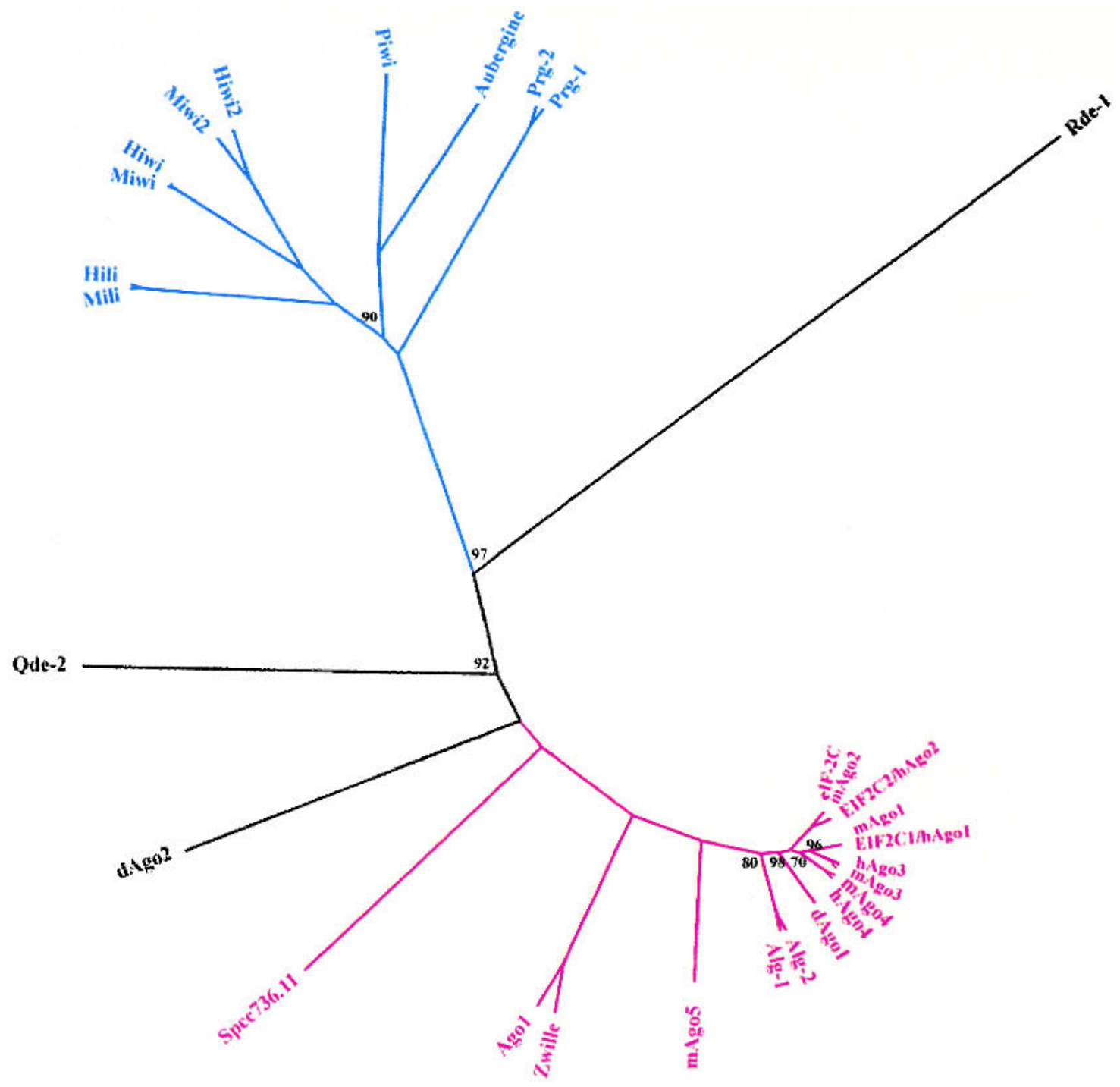

0.1

Figure 1. Phylogenetic tree of Argonaute proteins. Ago subfamily indicated in red, Piwi subfamily in blue, orphans in black. Multiple alignment was done by ClustalW (version 1.74). PHYLIP95 was used to do bootstrap analysis, protein distance calculation (using Dayhoff PAM distance), and tree construction. Bootstrap percentages are indicated at each fork where the percentage is not 100 . Accession nos. are: NP_510322, ALG-1; NP_493837, ALG-2; AAD40098, ZWILLE; AAD38655, aubergine/sting; JC6569, rabbit eIF-2C; CAA98113, Prg-1; AAB37734, Prg-2; AAF06159, RDE-1; AAF43641, QDE2; AAC18440, AGO1; NP_523734, dAgo1; NP_476875, piwi; AAF49619 plus additional N-terminal sequence from Hammond et al. (2001a), dAgo2; T41568, SPCC736.11; AY135687, mAgo1; AY135688, mAgo2; AY135689, mAgo3; AY135690, mAgo4; AY135691, mAgo5; AY135692, Miwi2; NP_067283, MILI; NP_067286, MIWI; XP_050334, hAgo2/EIF2C2; XP_029051, hAgo3; XP_029053, hAgo1/EIF2C1; BAB13393, hAgo4; AAH25995, HILI; AAK92281, HIWI; and AAH31060, Hiwi2.

tional gene silencing (PTGS) in plants and quelling in fungi. These genes include Caenorhabditis elegans rde-1 (Tabara et al. 1999), Arabidopsis AGO1 (Fagard et al. 2000), and Neurospora QDE2 (Cogoni and Macino 1997). The list of silencing phenomena, in which Argonaute proteins are involved, also includes transcriptional gene silencing mediated at the level of chromatin. For example, in Saccharomyces pombe, Volpe and colleagues (2002) recently demonstrated that disruption of components of the RNAi machinery, including the genes en- coding S. pombe's single Argonaute, Dicer, and RNAdependent RNA polymerase homologs, leads to loss of silencing of heterochromatic repeats at the centromere. The Drosophila piwi mutation also affects transcriptional gene silencing (Pal-Bhadra et al. 2002). In addition, a Tetrahymena piwi-related gene, TWI1, is required for DNA elimination, an essential step in the maturation of the transcriptionally active macronucleus. Remarkably, this process appears to involve small, 228 -nt RNAs (Mochizuki et al. 2002). 
Still other Argonaute proteins have been implicated in developmental control, and recent studies suggest that silencing and developmental functions may be linked (see below). However, in some cases, genetic studies have indicated exclusive roles in either silencing or developmental control (Morel et al. 2002), keeping open the possibility that Argonaute proteins may exert some of their biological functions through processes unrelated to RNAi.

Of the ten Argonaute proteins in Arabidopsis, two have been extensively studied. These proteins, AGO1 and ZWILLE, serve as an example of Argonaute proteins with some degree of functional overlap that nevertheless seem to have preferential roles and/or targets in the cell. AGO1, which is expressed throughout the plant at all stages of development, was first isolated as a mutant that pleiotropically affects general plant architecture. ago1 mutants exhibit numerous phenotypic abnormalities such as radialized leaves, abnormal infertile flowers with filamentous structures resembling small squid (thus the name Argonaute), and in some cases shoot apical meristem defects (Bohmert et al. 1998; Lynn et al. 1999). It should be noted that mutants affecting the shoot apical meristem, around which leaves are initiated and patterned, are known to produce abnormal leaf phenotypes (Medford et al. 1992). AGO1 was subsequently shown to be necessary for post-transcriptional silencing of transgenes as well as for cosuppression of transgenes and their corresponding homologous endogenous genes (Fagard et al. 2000). The second well-characterized Arabidopsis gene, PINHEAD/ZWILLE, plays a critical role in maintaining undifferentiated stem cells in the shoot apical meristem (Moussian et al. 1998; see below for further discussion). In addition, its low-level expression in the vasculature and the adaxial domain (upper surface) of the leaf leads to other pleiotropic defects (Lynn et al. 1999).

While ago1 and zwille have shoot apical meristem and other defects in common, the zwille defect is more obvious in the meristem, whereas the ago1 defect manifests itself more severely in general plant architecture, possibly indicating preferential roles for each protein. The ago1/zwille double mutant exhibits a more severe phenotype than does either mutation alone, and severity of the phenotype of each mutant depends on the copy number of the other gene (Lynn et al. 1999). Fertile hypomorphic ago1 mutants were isolated, which were impaired in PTGS and viral resistance but developmentally nearly normal. In contrast, ZWILLE was found not to participate in PTGS at all (Morel et al. 2002). Together, these observations indicate that AGO1 and ZWILLE likely act in redundant pathways, and that one can compensate, at least to some extent, for the loss of the other. However, these data also suggest that AGO1 and ZWILLE may each have distinct roles.

The same can be said for the Argonaute family in $C$. elegans, in which some members are involved in RNAi, and others in development. Mutants of the best characterized C. elegans Argonaute, rde-1, are strongly resistant to RNAi but are developmentally normal (Tabara et al. 1999). Two other family members, alg-1 and alg-2, functionally overlap and show strong developmental phenotypes, but are dispensable for RNAi in the soma (Cikaluk et al. 1999; Grishok et al. 2001).

Drosophila contains four characterized Argonaute proteins (Piwi, Aubergine, dAgo1, and dAgo2) plus one predicted from genomic DNA (dAgo3). piwi, aubergine, $d A g o 1$, and $d A g o 2$ have been implicated in RNAi-like silencing phenomena. $d A g o 1$ was shown to be required for efficient RNAi in Drosophila embryos, with its particular function lying downstream of Dicer in the pathway (Williams and Rubin 2002). piwi has been shown to be necessary for PTGS and some aspects of transcriptional gene silencing (Pal-Bhadra et al. 2002). Strains with mutations in piwi, aubergine/sting, and Ago1 also exhibit developmental phenotypes (see below).

aubergine (a.k.a. sting), which like piwi is expressed embryonically in the presumptive gonad, is characterized by mutations that affect germline development. Both piwi and aubergine are required in the female germline for oogenesis (Wilson et al. 1996; Cox et al. 1998; Schmidt et al. 1999). aubergine is also responsible for acting post-transcriptionally to maintain silencing of the $\mathrm{X}$-linked repetitive Stellate locus that is necessary for male fertility. The Stellate locus is silenced through a homology-dependent mechanism mediated by an RNA transcribed from paralogous repeats called suppressor of stellate, Su(Ste) (Aravin et al. 2001). In wild-type males, an unprocessed and therefore untranslated 8 -kb primary transcript is made from the Stellate locus. Mutations in aubergine result in the production of a mature $0.7-\mathrm{kb}$ Stellate mRNA from the tens to hundreds of copies of the locus. After translation, the resultant protein forms crystalline aggregates in spermatocytes and spermatids. This protein accumulation is coincident with a syndrome of meiotic defects, ultimately leading to sterility (Schmidt et al. 1999). Of note, although Aubergine clearly has the ability to affect silencing of tandemly repetitive genes such as Stellate, it is not involved in silencing of several retrotransposons and other genomic repeats in the germline (Aravin et al. 2001).

Interestingly, Aubergine localizes to the polar granules of the germline precursor pole cells along with Staufen, a double-stranded RNA binding protein, and Vasa, an RNA helicase. Aubergine also regulates translation of the germline determinant oskar through an undefined interaction with its 3' UTR (Wilson et al. 1996; Harris and Macdonald 2001). Aubergine was recently shown to be necessary for establishment of RNAi in oocytes in a maturation-dependent manner (Kennerdell et al. 2002). Interestingly, the competence for RNAi in oocytes is coincident with the translation of maternally loaded mRNAs, implying that these mRNAs must be translated before they are subject to degradation by RNAi.

The fourth Argonaute protein in Drosophila, dAgo2, has been shown to be necessary for RNAi as a component of the RISC complex (Hammond et al. 2001a). No $d A g o 2$ mutants have yet been reported, so it remains unknown whether $d A g O 2$ is involved in developmental control. 


\section{A subset of Argonaute proteins affect stem cell fate}

It is apparent that Argonaute proteins function in the development of varied tissues in diverse organisms, leading one to speculate as to a potential common denominator. There is increasing evidence to support the notion that such a common thread might be maintenance of stem cell character. The capacity of stem cells to selfrenew is crucial for the development and maintenance of a wide range of tissues, from germ cells, to hematopoietic progenitor cells, to the meristems of plants. The basic mechanisms that are responsible for establishing and maintaining stem cells are largely unknown and are likely to be comprised of both cell-autonomous and cellnonautonomous signals. Alterations in Argonaute protein function have been shown to affect stem cells in a variety of tissues in a disparate group of organisms, indicating that this protein family may be part of the most basic mechanisms governing stem cell fate.

Several Argonaute proteins appear to be involved in stem cell fate decisions, and mutations in these genes cause stem cells to lose their stem cell character and differentiate instead of undergoing self-renewing division. The best characterized Drosophila Argonaute family member, piwi, is required for the self-renewing, asymmetric division of both male and female germline stem cells (Cox et al. 1998). Larvae with mutations in piwi have normal numbers of germline stem cells, but adults lack them altogether. Instead, these animals contain differentiated cysts whose number approximately equals that of the germline stem cells from which they were derived. Consistent with a role in promoting the self-renewing division of germline stem cells, piwi is expressed in both the stem cells themselves and in surrounding somatic cells of the ovary. Analysis of genetic mosaics revealed that piwi expression in the ovary maintains stem cells through a somatic signaling mechanism (Cox et al. 1998). Overexpression in the soma also affects the initial establishment of stem cell character, expands the stem cell domain, increases the number of stem cells, and stimulates their division (Cox et al. 2000). Overexpression of piwi in the germline stem cells themselves also promotes division, indicating that piwi may also have a cell-autonomous function (Cox et al. 2000).

In C. elegans, reduced expression of the Argonaute family members that are most closely related, at the level of primary sequence, to piwi-that is, $P R G-1$ and $P R G$-2-produces germline defects very similar to those that result from piwi mutations in the fly. These include reduced proliferation of stem cells and a decrease in fertility (Cox et al. 1998). The forgoing observations indicate that piwi family members may have evolutionarily conserved roles in germline stem cell maintenance.

Drosophila Argonaute1 is essential for normal development, particularly in the nervous system. Mutations in $d A g o 1$ cause embryonic lethality marked by a severe decrease in all types of neurons and glial cells, a phenotype Kataoka et al. (2001) suggest may arise from a defect in cell cycle progression or cell survival. In accord with their assertions, generically reduced cell numbers are consistent with precocious quiescence of neuronal precursor cells, but numerous other potential causes cannot be excluded (Kataoka et al. 2001).

Strengthening the proposed connection between Argonaute proteins and stem cell fate, the developmental phenotypes of Arabidopsis ago1 and zwille mutants can be traced to a regulatory role involving stem cells in the shoot apical meristem. Shoot apical meristems contain pluripotent cells analogous to animal stem cells that are responsible for continually generating tissues and organs throughout the life of the plant. The organization of a shoot apical meristem is such that lateral organs are initiated at the periphery of a central zone of stem cells. These, when maintained properly in an undifferentiated state, provide a pool of pluripotent cells for future organogenesis (for review, see Fletcher 2002).

ZWILLE plays a critical role in maintaining undifferentiated stem cells in the shoot apical meristem during the transition from embryonic development to postembryonic organ formation (Moussian et al. 1998). Consistent with differentiation of the apex, zwille, like ago1, exhibits reduced expression of SHOOT MERISTEM$L E S S$, a homeobox gene that is required for initiation and maintenance of meristems (Moussian et al. 1998). The shoot apical meristems of zwille mutants, although initiated correctly, do not undergo asymmetric divisions that give rise to repetitive organs along the vertical axis of the plant. Instead, the stem cells in the central zone of the meristem lose their stem cell character and terminally differentiate, often forming a single central organ in place of the meristem (McConnell 1995; Moussian et al. 1998; Lynn et al. 1999). Homozygous mutants of some ago1 alleles exhibit a similar phenotype, with partial penetrance, producing a single, radially symmetric pin-like organ that is also thought to arise from premature differentiation of the shoot apical meristem (Lynn et al. 1999).

There is reason to believe that the connection between Argonaute family members and stem cell character might also exist in mammals. Human Hiwi is expressed in primitive hematopoietic progenitors $\left(\mathrm{CD} 34^{+}\right.$bone marrow stem cells) but not in more differentiated hematopoietic lineages. In fact, loss of Hiwi expression correlates with differentiation (Sharma et al. 2001). However, this finding must be viewed as suggestive since only a correlation was provided.

Additional correlative support for the involvement of Argonaute family proteins in regulating stem cell character comes from several connections that have arisen between Argonaute proteins and the Wingless/Wnt and Hedgehog pathways. These reciprocal signaling pathways control cell fate and developmental patterning in a diverse set of organisms, ranging from Drosophila to mammals. Particularly, dAgo1 was isolated in a screen for regulators of the Wingless signal transduction pathway. $d A g o 1$ overexpression rescued a wingless-like phenotype that was caused by sequestering the transcriptional activator Armadillo in the cytoplasm (Kataoka et al. 2001). However, loss of Drosophila ago1 did not give the segment polarity phenotypes that would be expected 
if Ago1 were actually a bona fide component of the Wingless pathway (Kataoka et al. 2001). Thus, Agol probably acts in a parallel pathway. Similarly, Piwi may act in parallel with Hedgehog, since Hedgehog overexpression in the ovary can compensate for lack of Piwi (King et al. 2001). This is consistent with the demonstration that Hedgehog acts as a somatic and germline stem cell-promoting factor (King et al. 2001; Zhang and Kalderon 2001).

Interestingly, a possible connection between Wingless signaling and an Argonaute protein may also exist in humans. The human EIF2C1/hAgo1 gene is often lost in Wilms' tumors of the kidney (see below), which are associated with activating mutations of $\beta$-catenin, the vertebrate counterpart of Armadillo (Dome and Coppes 2002).

\section{Argonaute proteins interact with stRNAs: the root of developmental phenotypes?}

Argonaute proteins in Drosophila, humans, and C. elegans have been shown to interact, genetically or biochemically, with small RNAs, now known generically as microRNAs or miRNAs (Grishok et al. 2001; Hutvagner et al. 2001; Ketting et al. 2001; Knight and Bass 2001; Hutvagner and Zamore 2002; Mourelatos et al. 2002). The founding members of this class are the small temporal RNAs, stRNAs, that regulate developmental transitions in C. elegans by controlling the expression of protein-coding genes. stRNAs and their relatives could, therefore, be the key to explaining the pleiotropic developmental phenotypes of Argonaute mutants in diverse organisms. stRNAs are noncoding RNAs that are synthesized as 60-70-nt precursors. These pre-stRNAs are processed into $\sim 21$-nt RNAs that bind complementary sites in the 3' UTRs of target genes, inhibiting their expression at the level of protein synthesis (for review on stRNAs, see Banerjee and Slack 2002).

There are several lines of evidence demonstrating that the developmental pathways regulated by stRNAs intersect with components of the RNAi pathway, although the full extent of the overlap has yet to be determined. The most compelling evidence comes from genetic studies in C. elegans. Particularly, Dicer (DCR-1) and the Argonaute proteins ALG-1 and ALG-2 have been implicated in processing of two well characterized stRNAs, let-7 and lin-4. The precursor forms of these genes accumulate in $d c r-1$ and alg-1/alg-2 knockouts (Grishok et al. 2001; Ketting et al. 2001; Knight and Bass 2001). There is also reason to speculate that the Drosophila ago1 neuronal defects could result from miRNA-mediated regulation, as several miRNAs with complementarity to negative regulatory elements in the 3'UTRs of genes that regulate neuronal development have been identified (Lai 2002). Furthermore, Dicer cleaves stRNA precursors in HeLa cells and in Drosophila embryo lysates (Hutvagner et al. 2001; Ketting et al. 2001). Finally, Drosophila Ago1 and Ago2 coimmunoprecipitate both siRNAs and miRNAs (Caudy et al., 2002), and human EIF2C2/
hAgo2 is a constituent of a complex from which $40 \mathrm{miR}$ NAs have been cloned (Mourelatos et al. 2002).

The stRNAs were originally identified as heterochronic genes in C. elegans affecting the transitions between stages in the life cycle by initiating a temporal cascade of regulatory genes that are responsible for developmental patterning (Lee et al. 1993; Feinbaum and Ambros 1999). miRNAs were also discovered in plants, including Arabidopsis and rice (Reinhart et al. 2000). It is therefore tempting to speculate that alterations in miRNA metabolism might underlie the developmental defects of the Arabidopsis ago1 and zwille mutations. However, it is important to note that AGO1 and ZWILLE may respond to signals distinct from those provided by miRNAs, and that miRNAs, as a class, might have quite diverse roles in regulating gene expression. This notion is supported by the finding that miRNAs represent a potentially large class of small RNAs, virtually all of which have unknown functions (Lagos-Quintana et al. 2001; Lau et al. 2001; Lee and Ambros 2001; Mourelatos et al. 2002).

\section{Argonaute proteins: fate determinants for processed RNAs?}

In contrast to double-stranded siRNAs, which are processed by Dicer from perfectly paired duplexes, at least some miRNAs are processed from imperfectly paired hairpin loops to form single-stranded 21-24-nt products (for review, see Banerjee and Slack 2002). Given the emerging genetic and biochemical studies of siRNA- and miRNA-containing complexes, one may now speculate on potential roles of Argonaute family proteins in specifying the fate of these two separate classes of RNAs processed by Dicer. Specifically, one is led to wonder how one enzyme processes structurally diverse triggers to yield products destined for regulating gene expression through distinct modes (altering mRNA stability vs. protein synthesis). It has been proposed that interactions with specific Argonaute family proteins may shepherd the different types of silencing triggers into appropriate complexes. Perhaps consistent with this notion, evidence is mounting which supports roles for Argonaute proteins at multiple steps in the RNAi mechanism.

In C. elegans, genetic analyses suggested that RDE-1 is required for the initiation of RNAi with injected dsRNAs (Grishok et al. 2000). Subsequently, biochemical analysis of RDE-1 complexes produced data consistent with this interpretation. Indeed, RDE-1 exists in a stable complex with Dicer and with several additional proteins, including a small dsRNA-binding protein, RDE-4, an RNA-dependent RNA polymerase homolog (RDE-9), and a DExH box helicase (Tabara et al. 2002). This has led to a model in which dsRNAs are processed to siRNAs through the action of this Argonaute-containing complex. Additional support for an initiator role also comes from studies of other Argonaute family members. Specifically, alg-1 and alg-2 mutations cause a reduction in the amount of mature, processed stRNAs produced 
(Grishok et al. 2001), and piwi mutant flies also show a decrease in siRNA production (Pal-Bhadra et al. 2002).

In some cases (particularly with alg-1 and alg-2), loss of Dicer products could result not from a defect in processing, but from reduced stability of the processed products because of their failure to enter effector complexes. Substantial evidence does exist for a role of Argonaute proteins at the effector step of RNAi. As mentioned above, Drosophila Ago2 is a component of RISC (Hammond et al. 2001a). Argonaute mutants in C. elegans (rde-1, under some circumstances), Drosophila (ago1), and Neurospora (qde-2) still produce siRNAs, indicating that some Argonautes are not required for production of the interfering agent (Parrish and Fire 2001; Catalanotto et al. 2002; Williams and Rubin 2002).

At both initiator and effector steps of RNAi, it is unclear whether Argonaute proteins exist in complexes that recognize all trigger types, or in discrete complexes, which preferentially recognize either siRNAs or miRNAs. Hutvagner and Zamore (2002) demonstrated that both miRNAs and siRNAs are associated with a single RNAi enzyme complex and that miRNAs can even direct cleavage of perfectly base-paired substrates. The Hutvagner and Zamore (2002) study suggests that the degree of complementarity between small RNA and substrate, and not incorporation into a discrete complex with a discrete Argonaute protein, determines its function. In this situation, the wide range of mutant phenotypes of these biochemically interchangable proteins might be explained by tissue specificity or developmentally regulated expression. However, another possibility is that, although both trigger types can be incorporated into the same complex, Argonaute proteins are preferentially associated with certain triggers. This might explain the differential requirements for various Argonaute proteins in development and RNAi functions in several organisms. Recent experiments have demonstrated that miRNAs coimmunoprecipitate and cofractionate mainly with Drosophila Agol in a complex distinct from RISC. However, miRNAs and siRNAs are found, to some extent, in both dAgol and dAgo 2 complexes (Caudy et al. 2002), indicating that any differential association is, at best, preferential and certainly not absolute.

Clearly, much additional work is necessary to decipher the many roles played by Argonaute family proteins in the RNAi pathway and the degree to which individual family members determine the outcome of the interaction between a small RNA and its cognate substrate.

\section{Mammalian Argonaute proteins}

The diversity of Argonaute family members in many species is consistent with their playing distinct or perhaps tissue-specific roles. An understanding of the biology of this multiprotein family will undoubtedly derive from genetic and biochemical studies in many model systems, including mammals. Although several mammalian Argonaute proteins have been identified, relatively little is known about their functions. The first mammalian Argonaute protein to be studied in any de- tail was rabbit eIF2C, which was identified as a component of a protein fraction that enhanced translation. However, it should be noted that no solid evidence exists for this protein playing a role in translation (Zou et al. 1998). Human EIF2C1/hAgo1, alternatively known as GERp95 for its association with Golgi and endoplasmic reticulum (ER; Cikaluk et al. 1999), was also identified in a screen for genes involved in Wilms' tumors (see below). Human EIF2C2/hAgo2 was recently shown to reside in a complex with Gemin3, an RNA helicase that joins the growing number of helicases implicated in RNAi, and Gemin4, a protein of unknown function (Mourelatos et al. 2002). Notably, that study also demonstrated an interaction between EIF2C2 and mature miRNAs. Hutvagner and Zamore (2002) solidified the link between this complex and RNAi by showing that it can cleave substrates that are homologous to its constituent miRNAs. Thus the EIF2C2/Gemin3/ Gemin4 complex may indeed represent mammalian RISC.

Two mouse homologs of Piwi, Miwi and Mili (for Miwi-like), have been examined in some detail and, although they differ in several aspects from Drosophila piwi, appear to be involved in regulation of spermatogenesis and primordial germ cell production. Presently, it is difficult to say whether one mouse Piwi family member most closely mimics the role of Drosophila Piwi or whether each performs a specialized subset of Piwi functions in the mouse. Miwi is not expressed in primordial germ cells of the embryo or in female gonads. However, it is detected in male gonads upon initiation of spermatogenesis two weeks after birth, when it is expressed in spermatocytes and spermatids (Kuramochi-Miyagawa et al. 2001; Deng and Lin 2002). Interestingly, in accordance with this protein expression pattern, spermatogenesis in miwi-null mice is arrested at the beginning of the round spermatid stage, well past the critical self-renewing divisions of germline stem cells (Deng and Lin 2002). Miwi, which binds to ribohomopolymers, also coimmunoprecipitates mRNAs corresponding to target genes of CREM, a master regulator of spermiogenesis. These target genes are downregulated in miwi-null mice, possibly indicating a role for Miwi in stabilization of these mRNAs that could be similar to the translational enhancement by Aubergine in flies. Interestingly, the miwi-null phenotype shows a more uniform and slightly earlier arrest of spermatogenesis than is caused by CREM mutations. Thus, these data define Miwi as a master switch in spermiogenesis, identifying the first known biological function of any mammalian Argonaute protein.

Mili, on the other hand, is expressed in primordial germ cells of embryos of both sexes from embryonic day 12.5 onward. Mili expression remains in males until the adult spermatocyte stage, and expression in females shows a marked decrease between birth and ovary development (Kuramochi-Miyagawa et al. 2001). Although a specific role in primordial germ cells has yet to be demonstrated, expression in these cell types may be a clue to a role for Mili that more closely mimics that of piwi. 


\section{Argonaute proteins and human disease}

Three closely related Argonaute family members (hAgo3, EIF2C1/hAgo1, and hAgo4) reside in tandem on chromosome 1 in human (the orthologous genes are in the same orientation on chromosome 4 in mouse). This region, 1p34-35, is often lost in Wilms' tumors (Koesters et al. 1999; Dome and Coppes 2002). Wilms' tumors are hypothesized to be caused by defects in embryonic kidney development that disturb the capacity of metanephrogenic precursor cells to differentiate. This permits primitive renal tissue to persist inappropriately and may ultimately lead to malignancy. Notably, EIF2C1/hAgo1 is expressed at low to medium levels in most tissues, but its expression is particularly high in embryonic kidney and lung. EIF2C1 levels are also increased in tumors that lack the Wilm's tumor suppressor gene WT1.

The human Piwi homolog, Hiwi, is located on chromosome 12q24.33. This locus displays linkage to the development of testicular germ cell tumors (Skotheim et al. 2001; Summersgill et al. 2001). In addition, Hiwi overexpression has been found in seminomas, tumors derived from embryonic germ cells that maintain stem cell character, suggesting a role for Hiwi reminiscent of the role of Drosophila Piwi in cell-autonomously driving stem cell division (Qiao et al. 2002). Furthermore, loss of the region of chromosome 12 containing Hiwi has been correlated with ambiguous genitalia and hypogonadism (Sathya et al. 1999). In at least two such studies, the lack of proper secondary sexual characteristics was attributed to primary testicular atrophy (Angulo et al. 1984; Sathya et al. 1999). Based on this information and the role of
Piwi-related genes in Drosophila and C. elegans germline development, there is a strong possibility that Hiwi is one of the loci that contribute to this phenotype.

\section{Bioinformatic analysis of mouse and human Argonaute proteins}

We have determined the chromosomal locations, gene structures, predicted cDNAs and protein sequences, as well as the ortholog and paralog relationships among the Argonaute genes in human and in mouse. Each Argonaute protein, of which there are seven in human and eight in mouse, has both a C-terminal PIWI domain and an N-terminal PAZ domain. Both mouse and human have three Argonaute proteins that fall in the Piwi subfamily, which most closely resembles Drosophila Piwi. Humans have four genes that fall into the Agol subfamily, most closely resembling Arabidopsis AGO1. Mouse has one additional Ago1 family gene resulting in a total of five. This additional gene, $m A g o 5$, may have an orthologous human gene that has not yet become apparent in the current human GoldenPath assembly (June 2002 freeze in http://genome.ucsc.edu). This issue should be resolved as the human genome sequencing and annotation become more complete.

Figure 2 shows the high degree of conservation between all 15 mouse and human Argonaute proteins in the regions corresponding to the PAZ and PIWI domains. In general, PAZ domains tend to be less conserved than PIWI domains when comparing Argonaute proteins across species (data not shown), and indeed differences

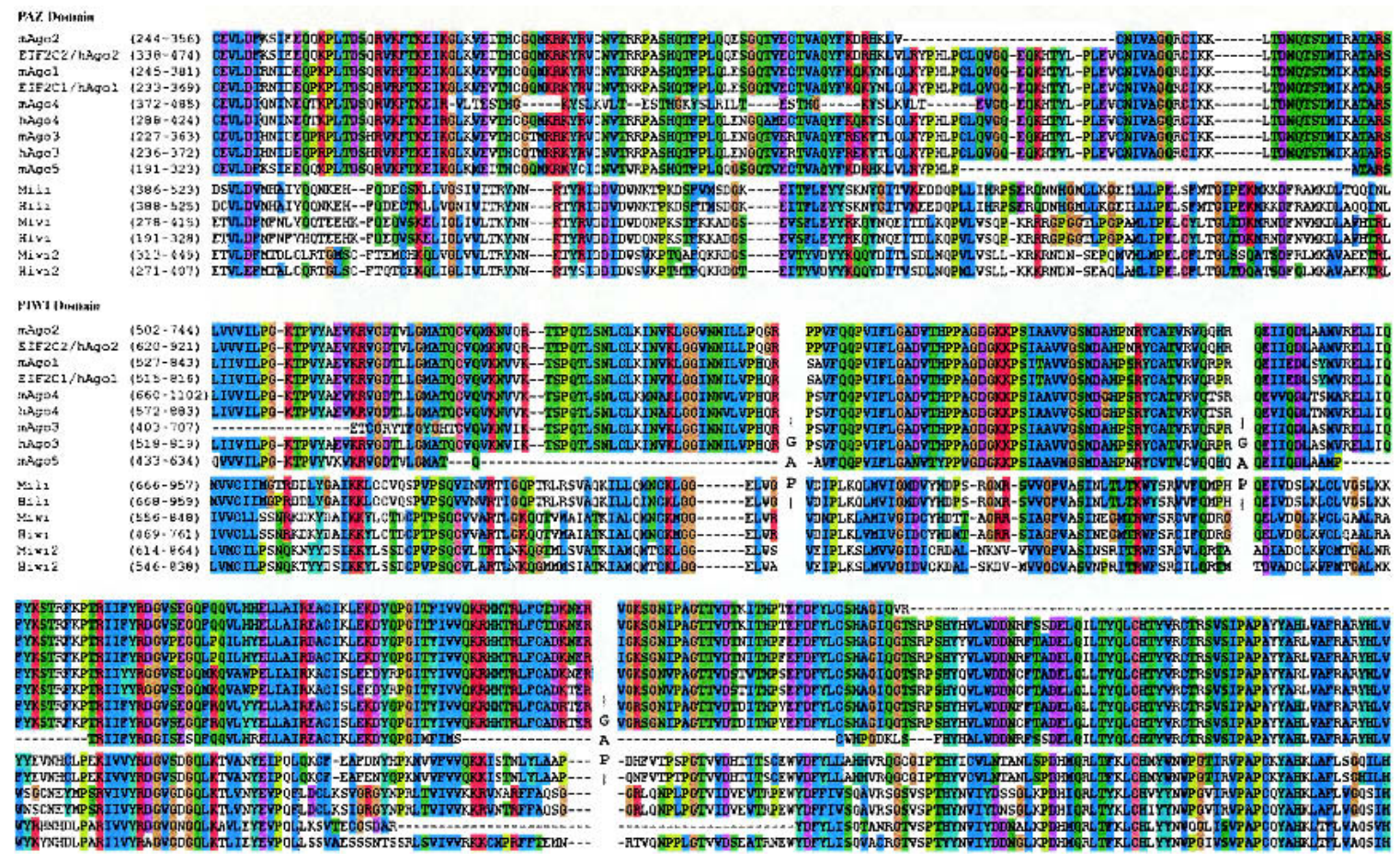

Figure 2. Alignment of mouse and human Argonaute PAZ and PIWI domains. Numbers in parentheses indicate the location of the motif in the amino acid sequence. Residues are color-coded as follows: orange, GPST; red, HKR; blue, FWY; green, ILMV; white, others. 
can be seen even between the two subfamilies of Argonaute proteins in mouse and human. In contrast, the PIWI domain is highly conserved between subfamilies (Fig. 2). Overall, the Piwi family in mouse shares an average of $38 \%$ identity within the subgroup, but Miwi and Hiwi are $86 \%$ identical. The Ago1 subfamily is more closely related $151 \%$ identical in mouse and $74 \%$ identical in human), whereas some orthologous genes are $>92 \%$ identical.

\section{Summary}

In conclusion, Argonaute proteins comprise a highly conserved protein family that is involved in a variety of RNA silencing phenomena in a diverse set of organisms. Perhaps through RNAi-related pathways or possibly also through distinct mechanisms, Argonaute family members have a variety of biological roles. These include regulation of development and determination of stemcell character. Through an ever-advancing understanding of the biochemistry and genetics of the Argonaute family, we may ultimately reveal the reach of these pathways into the basic biological mechanisms that underlie a variety of human diseases.

\section{Acknowledgments}

Data in Figures 1 and 2 were generated using, in part, information from the Celera Genome Database. M.C. is supported by a predoctoral fellowship from the U.S. Army Breast Cancer Research Program (DAMD-17-02-1-0343). G.J.H. is supported by an Innovator Award from the U.S. Army Breast Cancer Research Program and is a Rita Allen Foundation Scholar. This work was supported in part by grants from the NIH (HG01696, CA81152 to M.Q.Z. and RO1-GM62534 to G.J.H.).

\section{References}

Angulo, M.A., Castro-Magana, M., Sherman, J., Collipp, P.J., Milson, J., Trunca, C., and Derenoncourt, A.N. 1984. Endocrine abnormalities in a patient with partial trisomy 4q. $J$. Med. Genet. 21: 303-307.

Aravin, A.A., Naumova, N.M., Tulin, A.V., Vagin, V.V., Rozovsky, Y.M., and Gvozdev, V.A. 2001. Double-stranded RNA-mediated silencing of genomic tandem repeats and transposable elements in the D. melanogaster germline. Curr. Biol. 11: 1017-1027.

Banerjee, D. and Slack, F. 2002. Control of developmental timing by small temporal RNAs: A paradigm for RNA-mediated regulation of gene expression. Bioessays 24: 119-129.

Bernstein, E., Caudy, A.A., Hammond, S.M., and Hannon, G.J. 2001a. Role for a bidentate ribonuclease in the initiation step of RNA interference. Nature 409: 363-366.

Bernstein, E., Denli, A.M., and Hannon, G.J. 2001b. The rest is silence. RNA 7: 1509-1521.

Bohmert, K., Camus, I., Bellini, C., Bouchez, D., Caboche, M., and Benning, C. 1998. AGO1 defines a novel locus of Arabidopsis controlling leaf development. EMBO I. 17: 170-180.
Carthew, R.W. 2001. Gene silencing by double-stranded RNA. Curr. Opin. Cell. Biol. 13: 244-248.

Catalanotto, C., Azzalin, G., Macino, G., and Cogoni, C. 2002. Involvement of small RNAs and role of the qde genes in the gene silencing pathway in Neurospora. Genes \& Dev. 16: $790-795$.

Caudy, A.A., Myers, M., Hannon, G.J., and Hammond, S.M. 2002. Fragile X-related protein and VIG associate with the RNA interference machinery. Genes \& Dev. 16: 2491-2496.

Cerutti, L., Mian, N., and Bateman, A. 2000. Domains in gene silencing and cell differentiation proteins: The novel PAZ domain and redefinition of the Piwi domain. Trends Biochem. Sci. 25: 481-482.

Cikaluk, D.E., Tahbaz, N., Hendricks, L.C., DiMattia, G.E., Hansen, D., Pilgrim, D., and Hobman, T.C. 1999. GERp95, a membrane-associated protein that belongs to a family of proteins involved in stem cell differentiation. Mol. Biol. Cell 10: 3357-3372.

Cogoni, C. and Macino, G. 1997. Isolation of quelling-defective (qde) mutants impaired in posttranscriptional transgene-induced gene silencing in Neurospora crassa. Proc. Natl. Acad. Sci. 94: 10233-10238.

Cox, D.N., Chao, A., Baker, J., Chang, L., Qiao, D., and Lin, H. 1998. A novel class of evolutionarily conserved genes defined by piwi are essential for stem cell self-renewal. Genes \& Dev. 12: 3715-3727.

Cox, D.N., Chao, A., and Lin, H. 2000. piwi encodes a nucleoplasmic factor whose activity modulates the number and division rate of germline stem cells. Development 127: 503514.

Deng, W. and Lin, H. 2002. miwi, a murine homolog of piwi, encodes a cytoplasmic protein essential for spermatogenesis. Dev. Cell 2: 819-830.

Dome, J.S. and Coppes, M.J. 2002. Recent advances in Wilms tumor genetics. Curr. Opin. Pediatr. 14: 5-11.

Elbashir, S.M., Martinez, J., Patkaniowska, A., Lendeckel, W., and Tuschl, T. 2001. Functional anatomy of siRNAs for mediating efficient RNAi in Drosophila melanogaster embryo lysate. EMBO J. 20: 6877-6888.

Fagard, M., Boutet, S., Morel, J.B., Bellini, C., and Vaucheret, H. 2000. AGO1, QDE-2, and RDE-1 are related proteins required for post-transcriptional gene silencing in plants, quelling in fungi, and RNA interference in animals. Proc. Natl. Acad. Sci. 97: 11650-11654.

Feinbaum, R. and Ambros, V. 1999. The timing of lin-4 RNA accumulation controls the timing of postembryonic developmental events in Caenorhabditis elegans. Dev. Biol. 210: 87-95.

Fletcher, J.C. 2002. Coordination of cell proliferation and cell fate decisions in the angiosperm shoot apical meristem. Bioessays 24: 27-37.

Grishok, A., Tabara, H., and Mello, C.C. 2000. Genetic requirements for inheritance of RNAi in C. elegans. Science 287: 2494-2497.

Grishok, A., Pasquinelli, A.E., Conte, D., Li, N., Parrish, S., Ha, I., Baillie, D.L., Fire, A., Ruvkun, G., and Mello, C.C. 2001. Genes and mechanisms related to RNA interference regulate expression of the small temporal RNAs that control C. elegans developmental timing. Cell 106: 23-34.

Hamilton, A.J. and Baulcombe, D.C. 1999. A species of small antisense RNA in posttranscriptional gene silencing in plants [see comments]. Science 286: 950-952.

Hammond, S.M., Bernstein, E., Beach, D., and Hannon, G.J. 2000. An RNA-directed nuclease mediates post-transcriptional gene silencing in Drosophila cells. Nature 404: 293296. 
Hammond, S.M., Boettcher, S., Caudy, A.A., Kobayashi, R., and Hannon, G.J. 2001a. Argonaute2, a link between genetic and biochemical analyses of RNAi. Science 293: 1146-1150.

Hammond, S.M., Caudy, A.A., and Hannon, G.J. 2001b. Posttranscriptional gene silencing by double-stranded RNA. Nat. Rev. Genet. 2: 110-119.

Harris, A.N. and Macdonald, P.M. 2001. Aubergine encodes a Drosophila polar granule component required for pole cell formation and related to eIF2C. Development 128: 2823-2832.

Hutvagner, G. and Zamore, P.D. 2002. A MicroRNA in a multiple-turnover RNAi enzyme complex. Science 297: 20562060.

Hutvagner, G., McLachlan, J., Pasquinelli, A.E., Balint, E., Tuschl, T., and Zamore, P.D. 2001. A cellular function for the RNA-interference enzyme Dicer in the maturation of the let-7 small temporal RNA. Science 293: 834-838.

Kataoka, Y., Takeichi, M., and Uemura, T. 2001. Developmental roles and molecular characterization of a Drosophila homologue of Arabidopsis Argonaute1, the founder of a novel gene superfamily. Genes Cells 6: 313-325.

Kennerdell, J.R., Yamaguchi, S., and Carthew, R.W. 2002. RNAi is activated during Drosophila oocyte maturation in a manner dependent on aubergine and spindle-E. Genes \& Dev. 16: $1884-1889$.

Ketting, R.F., Fischer, S.E., Bernstein, E., Sijen, T., Hannon, G.J., and Plasterk, R.H. 2001. Dicer functions in RNA interference and in synthesis of small RNA involved in developmental timing in C. elegans. Genes \& Dev. 15: 2654-2659.

King, F.J., Szakmary, A., Cox, D.N., and Lin, H. 2001. Yb modulates the divisions of both germline and somatic stem cells through piwi- and hh-mediated mechanisms in the Drosophila ovary. Mol. Cell 7: 497-508.

Knight, S.W. and Bass, B.L. 2001. A role for the RNase III enzyme DCR-1 in RNA interference and germ line development in Caenorhabditis elegans. Science 293: 2269-2271.

Koesters, R., Adams, V., Betts, D., Moos, R., Schmid, M., Siermann, A., Hassam, S., Weitz, S., Lichter, P., Heitz, P.U., et al. 1999. Human eukaryotic initiation factor EIF2C1 gene: cDNA sequence, genomic organization, localization to chromosomal bands 1p34-p35, and expression. Genomics 61: $210-218$.

Kuramochi-Miyagawa, S., Kimura, T., Yomogida, K., Kuroiwa, A., Tadokoro, Y., Fujita, Y., Sato, M., Matsuda, Y., and Nakano, T. 2001. Two mouse piwi-related genes: miwi and mili. Mech. Dev. 108: 121-133.

Lagos-Quintana, M., Rauhut, R., Lendeckel, W., and Tuschl, T. 2001. Identification of novel genes coding for small expressed RNAs. Science 294: 853-858.

Lai, E.C. 2002. Micro RNAs are complementary to 3' UTR sequence motifs that mediate negative post-transcriptional regulation. Nat. Genet. 30: 363-364.

Lau, N.C., Lim, L.P., Weinstein, E.G., and Bartel, D.P. 2001. An abundant class of tiny RNAs with probable regulatory roles in Caenorhabditis elegans. Science 294: 858-862.

Lee, R.C. and Ambros, V. 2001. An extensive class of small RNAs in Caenorhabditis elegans. Science 294: 862-864.

Lee, R.C., Feinbaum, R.L., and Ambros, V. 1993. The C. elegans heterochronic gene lin-4 encodes small RNAs with antisense complementarity to lin-14. Cell 75: 843-854.

Lynn, K., Fernandez, A., Aida, M., Sedbrook, J., Tasaka, M., Masson, P., and Barton, M.K. 1999. The PINHEAD/ZWILLE gene acts pleiotropically in Arabidopsis development and has overlapping functions with the ARGONAUTE1 gene. Development 126: 469-481.

McConnell, J.R. 1995. Effect of mutations in the PINHEAD gene of Arabidopsis on the formation of shoot apical meristems. Dev. Genet. 16: 358-366.

Medford, J.I., Behringer, F.J., Callos, J.D., and Feldmann, K.A. 1992. Normal and abnormal development of the vegetative shoot apex. Plant Cell 4: 631-643.

Mochizuki, K., Fine, N.A., Fujisawa, T., and Gorovsky, M.A 2002. Analysis of a piwi-related gene implicates small RNAs in genome rearrangement in Tetrahymena. Cell 110: 689699.

Morel, J.B., Godon, C., Mourrain, P., Beclin, C., Boutet, S., Feuerbach, F., Proux, F., and Vaucheret, H. 2002. Fertile hypomorphic ARGONAUTE (agol) mutants impaired in posttranscriptional gene silencing and virus resistance. Plant Cell 14: 629-639.

Mourelatos, Z., Dostie, J., Paushkin, S., Sharma, A., Charroux, B., Abel, L., Rappsilber, J., Mann, M., and Dreyfuss, G. 2002. miRNPs: A novel class of ribonucleoproteins containing numerous microRNAs. Genes \& Dev. 16: 720-728.

Moussian, B., Schoof, H., Haecker, A., Jurgens, G., and Laux, T. 1998. Role of the ZWILLE gene in the regulation of central shoot meristem cell fate during Arabidopsis embryogenesis. EMBO J. 17: 1799-1809.

Pal-Bhadra, M., Bhadra, U., and Birchler, J.A. 2002. RNAi related mechanisms affect both transcriptional and posttranscriptional transgene silencing in Drosophila. Mol. Cell 9: 315327.

Parrish, S. and Fire, A. 2001. Distinct roles for RDE-1 and RDE-4 during RNA interference in Caenorhabditis elegans. RNA 7: 1397-1402.

Qiao, D., Zeeman, A.M., Deng, W., Looijenga, L.H., and Lin, H. 2002. Molecular characterization of hiwi, a human member of the piwi gene family whose overexpression is correlated to seminomas. Oncogene 21: 3988-3999.

Reinhart, B.J., Slack, F.J., Basson, M., Pasquinelli, A.E., Bettinger, J.C., Rougvie, A.E., Horvitz, H.R., and Ruvkun, G. 2000. The 21-nucleotide let-7 RNA regulates developmental timing in Caenorhabditis elegans. Nature 403: 901-906.

Sathya, P., Tomkins, D.J., Freeman, V., Paes, B., and Nowaczyk, M.J. 1999. De novo deletion 12q: Report of a patient with 12q24.31q24.33 deletion. Am. J. Med. Genet. 84: 116-119.

Schmidt, A., Palumbo, G., Bozzetti, M.P., Tritto, P., Pimpinelli, S., and Schafer, U. 1999. Genetic and molecular characterization of sting, a gene involved in crystal formation and meiotic drive in the male germ line of Drosophila melanogaster. Genetics 151: 749-760.

Sharma, A.K., Nelson, M.C., Brandt, J.E., Wessman, M., Mahmud, N., Weller, K.P., and Hoffman, R. 2001. Human CD34(+) stem cells express the hiwi gene, a human homologue of the Drosophila gene piwi. Blood 97: 426-434.

Skotheim, R.I., Kraggerud, S.M., Fossa, S.D., Stenwig, A.E., Gedde-Dahl Jr., T., Danielsen, H.E., Jakobsen, K.S., and Lothe, R.A. 2001. Familial/bilateral and sporadic testicular germ cell tumors show frequent genetic changes at loci with suggestive linkage evidence. Neoplasia 3: 196-203.

Summersgill, B., Osin, P., Lu, Y.J., Huddart, R., and Shipley, J. 2001. Chromosomal imbalances associated with carcinoma in situ and associated testicular germ cell tumours of adolescents and adults. Br. J. Cancer 85: 213-220.

Tabara, H., Sarkissian, M., Kelly, W.G., Fleenor, J., Grishok, A., Timmons, L., Fire, A., and Mello, C.C. 1999. The rde-1 gene, RNA interference, and transposon silencing in C. elegans. Cell 99: 123-132.

Tabara, H., Yigit, E., Siomi, H., and Mello, C.C. 2002. The dsRNA binding protein RDE-4 interacts with RDE-1, DCR1, and a DExH-box helicase to direct RNAi in C. elegans. Cell 109: 861-871. 


\section{Carmell et al.}

Volpe, T., Kidner, C., Hall, I.M., Teng, G., Grewal, S.I., and Martienssen, R. 2002. Regulation of heterochromatic silencing and histone H3 lysine-9 methylation by RNAi. Science 22: 22 .

Williams, R.W. and Rubin, G.M. 2002. ARGONAUTE1 is required for efficient RNA interference in Drosophila embryos. Proc. Natl. Acad. Sci. 99: 6889-6894.

Wilson, J.E., Connell, J.E., and Macdonald, P.M. 1996. Aubergine enhances oskar translation in the Drosophila ovary. Development 122: 1631-1639.

Zamore, P.D., Tuschl, T., Sharp, P.A., and Bartel, D.P. 2000. RNAi: Double-stranded RNA directs the ATP-dependent cleavage of mRNA at 21 to 23 nucleotide intervals. Cell 101: 25-33.

Zhang, Y. and Kalderon, D. 2001. Hedgehog acts as a somatic stem cell factor in the Drosophila ovary. Nature 410: 599604.

Zou, C., Zhang, Z., Wu, S., and Osterman, J.C. 1998. Molecular cloning and characterization of a rabbit eIF2C protein. Gene 211: $187-194$. 


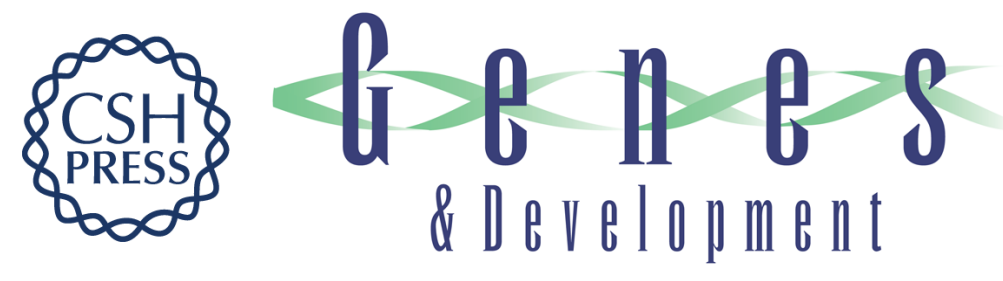

\section{The Argonaute family: tentacles that reach into RNAi, developmental control, stem cell maintenance, and tumorigenesis}

Michelle A. Carmell, Zhenyu Xuan, Michael Q. Zhang, et al.

Genes Dev. 2002, 16:

Access the most recent version at doi:10.1101/gad.1026102

References This article cites 65 articles, 33 of which can be accessed free at:

http://genesdev.cshlp.org/content/16/21/2733.full.html\#ref-list-1

License

Email Alerting Receive free email alerts when new articles cite this article - sign up in the box at the top Service right corner of the article or click here.

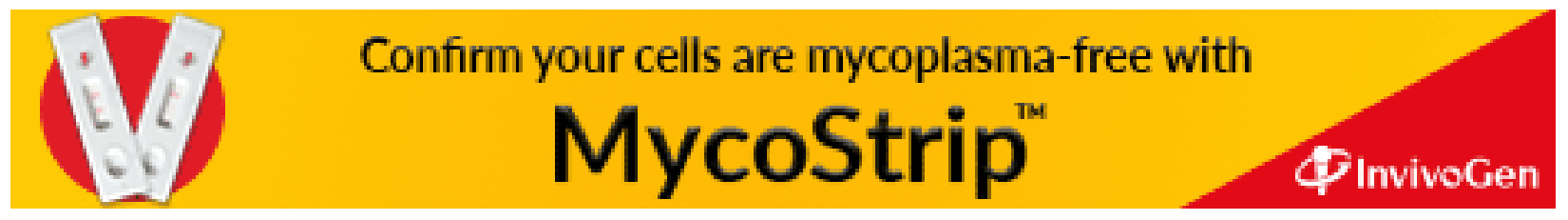

